\title{
Prevalence of Apical Periodontitis in patients with Multiple Myeloma
}

\author{
Ricardo E. Oñate-Sánchez, Sara Pérez-Díaz, Francisco J. Rodríguez-Lozano, Julia Guerrero-Gironés
}

Special Care Dentistry and Gerodontology Unit, School of Medicine and Dentistry, IMIB-Arrixaca, University of Murcia, Murcia, Spain

Correspondence:

School of Medicine and Dentistry, University of Murcia

Hospital Morales Meseguer $2 \mathrm{pl}$.

Av. Marqués de los Vélez, s/n

30008, Murcia, Spain

fcojavier@um.es

Received: 28/08/2019

Accepted: $17 / 02 / 2020$

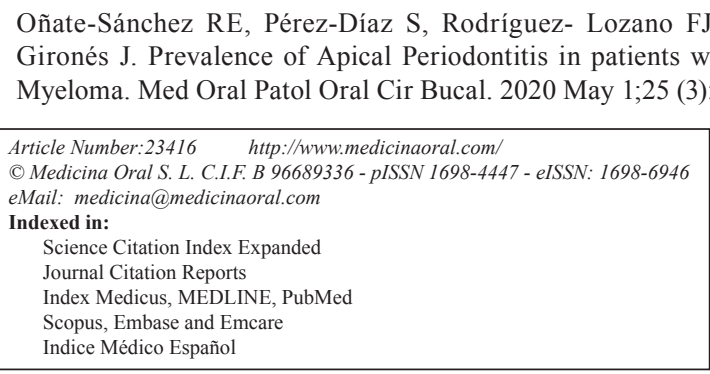

\begin{abstract}
Background: Previous reports have been analyzed the prevalence/association of apical periodontitis (AP) with systemic diseases. The present study aims to analyze the prevalence of healthy/diseased periapex and endodontic treatments in patients with Multiple Myeloma (MM) and compare the results with those of control subjects.

Material and Methods: Panoramic radiographs of 50 individuals with MM were evaluated and compared with 50 controls that were sex and age matched exactly with the diseased group. Radiographic analysis was performed by two experienced endodontists under standardized conditions. The periapical status (presence or not of AP) was assessed using the periapical index (PAI). Data included systemic health, technical quality of root fillings, total number of teeth, quality of restoration, and periapical status. Statistical evaluation of differences between groups used chi-squared tests and Fisher's exact tests.

Results: The prevalence of root canal-treated teeth was $10.11 \%$ in the MM group and $12.05 \%$ in the control group $(p=0.90)$. The average root canal-treated teeth in the test group was 2,34 and 2.48 in the control group, where the difference was statistically significant $(p=0.05$ ). AP in 1 or more teeth was found in $86 \%$ and in $78 \%$ of the patients in the MM and the control groups, respectively. When analyzed by subject, there was no statistically significant difference in the prevalence of AP $(p>0.72)$. Similarly there was also no statistically significant difference in the prevalence of PA ( $p=0.85$ ), when analyzed by tooth, AP was found in $63.2 \%$ and $62.9 \%$ in MM and control groups.

Conclusions: The presence of AP and endodontic treatment was not significantly different in individuals with MM compared with control subjects. Future studies are needed to elucidate and confirm the association between MM and AP.
\end{abstract}

Key words: Apical periodontitis, multiple myeloma, endodontics, root canal treatment, systemic disease. 


\section{Introduction}

Apical periodontitis (AP) is the inflammation and destruction of the periapical tissues as a consequence of a pulpal necrosis. It can also result from a pre-existing acute abscess or inadequate endodontic therapy $(1,2)$. AP is a fairly frequent condition that increases with age. It occurs in 34 to $61 \%$ of people and 2.8 to $4.2 \%$ of teeth in Europe (3). The AP treatment of choice is root canal treatment (RCT), which is estimated at around $41-59 \%$ of individuals and $2-6,4 \%$ of teeth, with radiographic evidence of persistent chronic AP in $24-65 \%$ of root filled teeth (4). Factors like nutrition or the existence of systemic pathology could influence the repair of periapical lesions. Compromised immune systems may play an important role in the development, progression and healing of AP (5). When dental and medical scientific communities have analyzed the relation between AP and systemic health (6), the results suggest an association between PA and diabetes mellitus (7), coronary heart disease (8), tobacco smoking (9) and other diseases like respiratory diseases (10) or osteoporosis in post menopause women (11).

Multiple myeloma (MM) accounts for $10 \%$ of neoplasms in bone marrow, the average age of individuals at the time of diagnosis being 65 years, with less than $15 \%$ of cases occurring below 50 years. Each year 3-5 cases per 100,000 people are diagnosed (12). The disease is primarily characterized by anemia, increased risk of infection, hypercalcemia, bone lesions and renal failure and it is the second most common hematological malignancy (13). MM interferes with the mechanisms of bone renewal causing its decalcification (osteolytic bone lesions) that usually cause intense bone pain and increase the risk of spontaneous fractures. The most common locations affected are the spine, ribs, pelvis and skull. Diagnosis of MM is made by a complete protein analysis in blood and urine, which allows the monoclonal component to be correctly characterized, while a bone marrow aspirate is used to confirm the presence of abnormal plasma cells in the bone marrow. In addition, the detection of bone lesions must be determined by a radiological study of the entire skeleton. The primary clinical manifestation of the disease will be found in the oral cavity in $14 \%$ of myeloma patients $(14,15)$. Maxillofacial manifestations of MM appear more frequently in the mandible than in the maxilla with an incidence of $8-15 \%(16-19)$ and they include soft-tissue amyloid deposits (20), external dental root resorption (21), hypesthetic or anesthetic sensation of the lower lip (Vicent symptom), pain, gingival enlargement, swelling, tooth loosening, osteolytic lesions and amyloidosis with macroglossia $(22,23)$. However, there have been no studies evaluating the association between MM and AP.

The purpose of this study was to evaluate the presence of apical periodontitis and endodontic treatments in pa- tients with myeloma multiple in comparison with subjects without myeloma multiple.

\section{Material and Methods}

- Data collection

A total of 220 patients with multiple myeloma were primarily included in the experimental group. These patients were referred to the School of Medicine and Dentistry (Universidad de Murcia, Spain) for an assessment of their oral health status during the years 2013-2015 . Patients were selected if they fulfilled the following inclusion criteria: 1) at least 18 years old; 2) having a complete medical and dental history, including panoramic radiographs of the maxilla and mandible; 3) the presence of at least one root canal treatment; 4) a follow-up radiograph taken a minimum of 1 year after the root canal filling. The following were used as exclusion criteria: 1) patients who refused to have an Xray examination; 2) patients with removed pieces that were previously endodontically treated at the moment of this study; and 3) patients with other malign or hematological diseases. The University of Murcia Committee on Research Involving Human Subjects of (ID: 1700/2017) approved the study, which was carried out in accordance with the principles outlined in the Helsinki Declaration i (as revised in 2000) on experimentation involving humans. Study subjects and control patients were asked to voluntarily participate in the study and gave their informed written consent.

Following inclusion/exclusion criteria, the experimental group finally comprised 50 individuals affected by multiple myeloma and the presence of root canal treatment. The controls were sex and age matched with the test group so that the same numbers of individuals without MM were included.

- Assessment of radiographs

Panoramic radiographs were taken of all the patients using Vatech Pax-400 (Vatech Co., Ltd., Gyeonggi-do, Korea) by an experienced radiographic technician. Radiographic analysis was performed by two experienced endodontists, who recorded and evaluated the following information for each patient: sex, age, number of teeth present, number and location of teeth endodontically treated, quality of root canal fillings, quality of coronal restoration, length of root canal filling and periapical status. The two examiners were calibrated based on the criteria and variants established before their evaluation. The results of all of teeth evaluations were recorded, and were categorized as root-filled teeth if they had been filled with radiopaque material in the root canal(s). The periapical status (presence of AP) was assessed using the periapical index (PAI). The roots were categorized as follows: 1, normal periapical structures; 2, small changes in bone structure: 3 , changes in bone structure with some mineral loss; 4 , periodontitis with a well-de- 
fined radiolucent area; and 5, severe periodontitis with exacerbating features. A score greater than 2 (PAI $\geq 3$ ) was considered to be a sign of periapical pathology. The worst score of all roots was taken to represent the PAI score for multirooted teeth.

Root canal fillings were judged adequate when all canals were obturated with no voids present and root fillings ended from 0-2 mm short of the radiographic apex. Multirooted teeth were categorized by the root of the most defective filling. Coronal restoration was classified as adequate when the restoration was radiographically intact and had no signs of overhangs, open margins or recurrent caries. Discrepant cases were resolved by joint discussion until consensus was achieved.

- Statistical analysis

All analyses were performed in an SPSS environment (Version 22.0; Inc., Chicago, IL, USA). The study of the outcomes was reported as frequency/percentage and compared between the groups using the Pearson chisquare test and Fisher's exact tests. A t test was used to determine whether or not the mean age between groups differed significantly. The level of significance adopted was $p<0.05$.

\section{Results}

Fifty patients (29 women and 21 men) with MM ranging in age from 35 to 84 years old $(63.38 \pm 10.585)$ were identified as eligible for this study (Table 1). The control group consisted of 50 subjects ( 28 women and 22 men) ranging from 38 to 84 years old $(63.44 \pm 12.188)$. The difference was not statistically significant $(p>0.05)$. The average number of teeth per patient in the test group and the control group were 23.14 and 20.58, respective- ly, where the difference being statistically significant $(p=0.02)$. The prevalence of root canal-treated teeth was $10.11 \%$ in the MM group and $12.05 \%$ in the control group $(p=0.90)$. The average number of root canaltreated teeth in the test group was 2,34 and 2.48 in control group, the difference being statistically significant $(p=0.05)$.

AP in one or more teeth was found in $86 \%$ and $78 \%$ of patients in the MM and control groups, respectively. When analyzed by subject, there was no statistically significant difference in the prevalence of AP $(p>0.72)$. Similarly, there was no statistically significant difference in the prevalence of PA $(p=0.85)$, when analyzed by tooth $(63.2 \%$ and $62.9 \%$ in MM and control groups, respectively).

In the MM group, $63 \%$ of the root canal-treated teeth had AP, and in the control group the percentage was $63 \%(p>0.85)$.

The number of teeth with adequate endodontic filling in the MM group represented $38 \%$ of the root canaltreated teeth, $60 \%$ of which had AP $(p=0,65)$. In the control individuals, teeth with adequate endodontic filling represented $76 \%$ of treated teeth, $65 \%$ of which had AP $(p=0.95)$.

In the $\mathrm{MM}$ and control groups respectively, $40 \%$ and $67 \%$ of teeth revealed adequate crown restoration, in MM group $69.6 \%$ of teeth had AP $(p=0.19)$, and in the control group $61 \%(p=0.56)$.

The number of teeth of adequate length in the MM group was equal to $63 \%$ of the root canal-treated teeth, $69 \%$ of which had AP $(p=0.88)$, while in the control group $67 \%$ of root canal-treated teeth had an adequate length but $65 \%$ of these had AP.

Table 1: Distribution of the Analyzed Variables in Patients with MM (Study Group) and Control Subjects.

\begin{tabular}{|c|c|c|c|}
\hline & Multiple Myeloma & Control & $P$ value \\
\hline Patients with RCT, n (\%) & 50(29 Female, 21Male) & 50 (28Female, 22Male) & .84 \\
\hline Patients with PAR, n (\%) & $43(86 \%)$ & $39(78 \%)$ & .72 \\
\hline Mean age $(\mathrm{y})$ & $63.38 \pm 10.58$ & $63.44 \pm 12.19$ & .98 \\
\hline Teeth & 1157 & 1029 & .02 \\
\hline RCT Teeth, n (\%) & $117(10.11)$ & $124(12.05)$ & .05 \\
\hline Teeth with PAR, n (\%) & $74(63)$ & $78(63)$ & 0.85 \\
\hline \multirow[t]{2}{*}{ RCT filling adequate (\%) } & $45(38)$ & $94(76)$ & \\
\hline & $\begin{array}{c}\text { With PAR } 27(60) \\
\text { Without PAR } 18(40) \\
\quad p=0.65\end{array}$ & $\begin{array}{c}\text { With PAR 61(65) } \\
\text { Without PAR 33(35) } \\
\quad p=0.95\end{array}$ & \\
\hline \multirow[t]{2}{*}{ Adequate crown rest. } & $46(40)$ & $83(67)$ & \\
\hline & $\begin{array}{c}\text { With PAR 32(69.6) } \\
\text { Without PAR 14(31.4) } \\
p=0.19\end{array}$ & $\begin{array}{c}\text { With PAR } 51(61) \\
\text { Without PAR 32(39) } \\
\quad p=0.56\end{array}$ & \\
\hline \multirow[t]{2}{*}{ Adequate length } & $74(63)$ & $83(67)$ & \\
\hline & $\begin{array}{c}\text { With PAR 51(69) } \\
\text { Without PAR 23(31) } \\
\quad p=0.88\end{array}$ & $\begin{array}{c}\text { With PAR 54(65) } \\
\text { Without PAR 29(35) } \\
\quad p=0.42\end{array}$ & \\
\hline
\end{tabular}




\section{Discussion}

Previous studies have reported the strong association between several systemic diseases and endodontic variables, such as apical periodontitis and the outcome of RCT, assessed as root filled teeth (RFT) with radiolucent periapical lesions (RPL) or non-retained RFT (24). The present study aimed to analyze the prevalence of healthy/diseased periapex and endodontic treatments in patients with MM and compare the same with control subjects (without MM).

Manifestations of Multiple Myeloma include osteolytic lesions or punched-out lesions on the jawbones and it may occur with a similar presentation to other cysts and odontogenic lesions. However, the osteolytic lesions are not usually located in periapical regions, but in the posterior teeth region, ramus, and condyle (25).

As regards evaluating endodontic variables using panoramic radiographs (PAN), this technique is a simple tool for diagnosing apical periodontitis because of its easy acquisition, low radiation dose, and panoramic views, as mentioned by Nardi et al. (26). Furthermore, two-dimensional imaging (PAN and periapical radiography) is commonly accepted as a method for the diagnosis of AP, while three-dimensional imaging (conebeam computed tomographic (CBCT)) represents a second evaluation step in some cases $(26,27)$.

Due to the immunosuppression status of these patients (28), inclusion and exclusion criteria were strictly applied and patients with other malign or hematological diseases were excluded, in order to reduce the effect of covariables. However, the lack of other studies evaluating the presence of AP lesions and endodontic treatments in MM patients must be regarded as the main limitation of this work.

Our data showed no statistically significant difference was observed between MM and control as regards the presence of apical periodontitis and the average size of the periapical lesions (Table 1). However, we observed poor crown restoration and root canal fillings in the Myeloma group. This may be due to the periodic lack of dental visits beacause they prioritize the medical problem, or to the age of the patients. In this respect, Persic Bukmir et al. (29) demonstrated that age was significantly correlated with poor periapical status, and it has already been defined as a risk indicator for AP (30). The same author confirmed that periodic dental visits to dental practitioners reduces the periapical disease ratio (29).

Other authors have postulated that the quality of the root canal fillings or the coronal restoration have a similar impact on the resolution of endodontic infections as the influence exerted by an impaired or modulated immune system (5). Such a correlation was not observed in our study, although a limitation might have been the fact that patients had been suffering from the disease for several years, and not all of them were immunocompromised at the same time. Indeed, the severity of any immunosuppression is another factor that has been reported (6).

\section{Conclusions}

The results of the present study showed that the presence of AP and endodontic treatment was not significantly different between individuals with MM and control subjects. More studies are needed to elucidate and confirm the association between MM and AP.

\section{References}

1. Chauhan N, Mittal S, Tewari S, Sen J, Laller K. Association of Apical Periodontitis with Cardiovascular Disease via Noninvasive Assessment of Endothelial Function and Subclinical Atherosclerosis. J Endod. 2019;45:681-90.

2. Skallsjo K, Johansson JE, Jonasson P, Hasseus B. Apical periodontitis as potential source of infection in patients with lymphoma treated with chemotherapy. Clin Oral Investig. 2020;24:133-40.

3. Nair PN. Pathogenesis of apical periodontitis and the causes of endodontic failures. Crit Rev Oral Biol Med. 2004;15:348-81.

4. Segura-Egea JJ, Martin-Gonzalez J, Castellanos-Cosano L. Endodontic medicine: connections between apical periodontitis and systemic diseases. Int Endod J. 2015;48:933-51.

5. Cotti E, Schirru E, Acquas E, Usai P. An overview on biologic medications and their possible role in apical periodontitis. J Endod. 2014;40:1902-11.

6. Laukkanen E, Vehkalahti MM, Kotiranta AK. Impact of systemic diseases and tooth-based factors on outcome of root canal treatment. Int Endod J. 2019; 52:1417-26.

7. Lopez-Lopez J, Jane-Salas E, Estrugo-Devesa A, Velasco-Ortega E, Martin-Gonzalez J, Segura-Egea JJ. Periapical and endodontic status of type 2 diabetic patients in Catalonia, Spain: a cross-sectional study. J Endod. 2011;37:598-601.

8. Singhal RK, Rai B. sTNF-R Levels: Apical Periodontitis Linked to Coronary Heart Disease. Open Access Maced J Med Sci. 2017;5:68-71. 9. Lopez-Lopez J, Jane-Salas E, Martin-Gonzalez J, CastellanosCosano L, Llamas-Carreras JM, Velasco-Ortega E, et al. Tobacco smoking and radiographic periapical status: a retrospective casecontrol study. J Endod. 2012;38:584-8.

10. Scannapieco FA, Bush RB, Paju S. Associations between periodontal disease and risk for nosocomial bacterial pneumonia and chronic obstructive pulmonary disease. A systematic review. Ann Periodontol. 2003;8:54-69.

11. Lopez-Lopez J, Castellanos-Cosano L, Estrugo-Devesa A, Gomez-Vaquero C, Velasco-Ortega E, Segura-Egea JJ. Radiolucent periapical lesions and bone mineral density in post-menopausal women. Gerodontology. 2015;32:195-201.

12. Kumar SK, Rajkumar SV. The multiple myelomas - current concepts in cytogenetic classification and therapy. Nat Rev Clin Oncol. 2018;15:409-21.

13. Liu P, Jin Y, Sattar H, Liu H, Xie W, Zhou F. Natural killer cell immunotherapy against multiple myeloma: Progress and possibilities. J Leukoc Biol. 2018;103:821-8.

14. Adeyemo TA, Adeyemo WL, Adediran A, Akinbami AJ, Akanmu AS. Orofacial manifestation of hematological disorders: hemato-oncologic and immuno-deficiency disorders. Indian J Dent Res. 2011;22:688-97.

15. Zhao XJ, Sun J, Wang YD, Wang L. Maxillary pain is the first indication of the presence of multiple myeloma: A case report. Mol Clin Oncol. 2014;2:59-64.

16. Cardoso RC, Gerngross PJ, Hofstede TM, Weber DM, Chambers MS. The multiple oral presentations of multiple myeloma. Support Care Cancer. 2014;22:259-67. 
17. Jain S, Kaur H, Kansal G, Gupta P. Multiple myeloma presenting as gingival hyperplasia. J Indian Soc Periodontol. 2013;17:391-3. 18. Stoopler ET, Vogl DT, Stadtmauer EA. Medical management update: multiple myeloma. Oral Surg Oral Med Oral Pathol Oral Radiol Endod. 2007;103:599-609.

19. Epstein JB, Voss NJ, Stevenson-Moore P. Maxillofacial manifestations of multiple myeloma. An unusual case and review of the literature. Oral Surg Oral Med Oral Pathol. 1984;57:267-71.

20. Viggor SF, Frezzini C, Farthing PM, Freeman CO, Yeoman $\mathrm{CM}$, Thornhill MH. Amyloidosis: an unusual case of persistent oral ulceration. Oral Surg Oral Med Oral Pathol Oral Radiol Endod. 2009;108:e46-50.

21. Troeltzsch M, Oduncu F, Mayr D, Ehrenfeld M, Pautke C, Otto S. Root resorption caused by jaw infiltration of multiple myeloma: report of a case and literature review. J Endod. 2014;40:1260-4.

22. Lee SH, Huang JJ, Pan WL, Chan CP. Gingival mass as the primary manifestation of multiple myeloma: report of two cases. Oral Surg Oral Med Oral Pathol Oral Radiol Endod. 1996;82:75-9.

23. Khurram SA, McPhaden A, Hislop WS, Hunter KD. Crystal storing histiocytosis of the tongue as the initial presentation of multiple myeloma. Oral Surg Oral Med Oral Pathol Oral Radiol Endod. 2011;111:494-6.

24. Segura-Egea JJ, Cabanillas-Balsera D, Jimenez-Sanchez MC, Martin-Gonzalez J. Endodontics and diabetes: association versus causation. Int Endod J. 2019;52:790-802.

25. Faria KM, Brandao TB, Silva WG, Pereira J, Neves FS, Alves $\mathrm{MC}$, et al. Panoramic and skull imaging may aid in the identification of multiple myeloma lesions. Med Oral Patol Oral Cir Bucal. 2018;23:e38-e43.

26. Nardi C, Calistri L, Grazzini G, Desideri I, Lorini C, Occhipinti $\mathrm{M}$, et al. Is Panoramic Radiography an Accurate Imaging Technique for the Detection of Endodontically Treated Asymptomatic Apical Periodontitis? J Endod. 2018;44:1500-8.

27. Nardi C, Calistri L, Pradella S, Desideri I, Lorini C, Colagrande S. Accuracy of Orthopantomography for Apical Periodontitis without Endodontic Treatment. J Endod. 2017;43:1640-6.

28. Braga-Diniz JM, Santa-Rosa CC, Martins RC, Silva M, Vieira LQ, Ribeiro Sobrinho AP. The need for endodontic treatment and systemic characteristics of hematopoietic stem cell transplantation patients. Braz Oral Res. 2017;31:e50.

29. Persic Bukmir R, Vidas J, Mance D, Pezelj-Ribaric S, Spalj $\mathrm{S}$, Brekalo Prso I. Socio-economic and health status as a predictor of apical periodontitis in adult patients in Croatia. Oral Dis. 2019;25:300-8.

30. Persic Bukmir R, Jurcevic Grgic M, Brumini G, Spalj S, PezeljRibaric S, Brekalo Prso I. Influence of tobacco smoking on dental periapical condition in a sample of Croatian adults. Wien Klin Wochenschr. 2016;128:260-5.

\section{Funding}

None declared.

Conflict of interest

None declared.

\section{Ethics}

The University of Murcia Committee on Research Involving Human Subjects of (ID: 1700/2017) approved the study. 